\title{
A space-time analysis of recurrent malnutrition-related hospitalisations in Kilifi, Kenya for children under-5 years
}

Kennedy Mwai Wambui and Eustasius Musenge*

\begin{abstract}
Background: Globally, malnutrition underlies $45 \%$ of under-5 s mortality, mainly from potentiating common infections such as diarrhoea and pneumonia. Malnutrition as a public health problem is not evenly disbursed because of disparities in food insecurity and health, and children commonly suffer recurrent episodes of opportunistic infections. We aimed to understand better the spatial and temporal structure of multiple paediatric hospital admissions associated with malnutrition-related illnesses. This paper aimed to investigate the spatialtemporal variations in malnutrition-related recurrent morbidity of children under-5 years from the Kilifi County in Kenya between 2002 and 2015.

Methods: The study included data from children under-5 years old who had more than one admission to a rural district hospital in Kenya within the Kilifi Health and Demographic Surveillance System (KHDSS). The primary outcome was a malnutrition-related admission, based on wasting (WHZ <-2) or nutritional oedema. Individual, household and environmental level covariates were examined as exposures. We first fitted a SARIMA model for the temporality, and the Moran's Index affirmed spatial clustering in malnutrition admissions. Kulldorf Statistics using SaTScan were applied to detect hotspots. Then, bivariate analysis was done using repeated values tabulation and analysis of covariance (ANCOVA). Inferential analysis was done using a mixed effect multivariable negative-binomial regression model, adjusting for spatiotemporal random effects.
\end{abstract}

Results: A total of 2821 children were admitted more than once, giving a total of 6375 admissions. Of these 6375 admissions, 1866 were malnutrition-related, and 3.9\% (109/2821) of the children with repeat admissions died. There was a seasonal pattern of re-admissions, peaking from May to July over the years. Hotspots were found in both the Northern and Southern areas of the KHDSS, while the areas near Kilifi Town were least affected. We found that disease severity was most likely associated with a malnutrition re-admission to the hospital.

Conclusion: Disease severity was strongly associated with admission with malnutrition but its effect reduced after adjusting for the spatial and temporal random effects. Adjusting for clustering in space and in time (spatialtemporal) in models helps to improve the understanding of recurrent hospitalisations involving malnutrition.

Keywords: Spatiotemporal analysis, Child malnutrition, Bayesian, Child mortality

\footnotetext{
* Correspondence: Eustasius.Musenge@wits.ac.za

Division of Epidemiology and Biostatistics, School of Public Health, Faculty of

Health Sciences, University of the Witwatersrand, Johannesburg, South Africa
}

(c) The Author(s). 2019 Open Access This article is distributed under the terms of the Creative Commons Attribution 4.0 International License (http://creativecommons.org/licenses/by/4.0/), which permits unrestricted use, distribution, and reproduction in any medium, provided you give appropriate credit to the original author(s) and the source, provide a link to the Creative Commons license, and indicate if changes were made. The Creative Commons Public Domain Dedication waiver (http://creativecommons.org/publicdomain/zero/1.0/) applies to the data made available in this article, unless otherwise stated. 


\section{Background}

Globally, malnutrition is a cause of $45 \%$ of childhood mortality, predominantly by potentiating morbidity due to common infections such as diarrhoea and pneumonia $[1,2]$. Sub-Saharan Africa and South Asia remain the areas with the highest prevalence of malnutrition [2]. In Sub-Saharan Africa, malnutrition is a leading cause of death among children in marginalised populations [2, 3]. It remains a significant contributor to in-patient morbidity and mortality among children in rural areas in Kenya, despite efforts to overcome malnutrition [4-6]. When various infections occur, malnutrition complicates their management and increases their case fatality [7]. The case-fatality ratios for hospitalised severely-malnourished children typically range from $12 \%$ to more than $30 \%[2,5,8]$. After discharge, an insufficient recovery period between illness episodes exacerbates malnutrition resulting in a vicious cycle. Malnutrition also majorly affects childhood development and increases the risk of non-communicable diseases and socioeconomic productivity in adulthood [1].

Undernutrition is a result of many determinants and not solely related to food insecurity. In Kenya, fundamental immediate and underlying causes of undernutrition have been identified to vary across the different age groups of 0-24 months and 25-59 months. Child's birth size, breastfeeding patterns and acute morbidity experience have more impact on the younger age group compared to the underlying factors affecting the older age group $[1,9,10]$. Morbidity which is one of the immediate factors leading to undernutrition, often resulting in longer and repeated hospital admissions. Morbidity is therefore associated with increased inpatient costs to families and sustains impoverishment [9].

Because malnutrition is due to a complex interaction between different components, it is difficult to understand in a way that helps extend preventive and treatment strategies from current vertical interventions $[1,11]$. Malnutrition has been reported to be spatially heterogeneous and also known to interact with various components of the environment, but little research has been done to integrate health, environmental and population data [12]. Up to now, a lack of detailed cohort and spatial data at homestead level had been a significant limitation for such spatial-temporal centred research of malnutrition in sub-Saharan Africa [11]. In this study, we use joint spatial-temporal models rather than mainly cross-sectional approaches [13] to give a different understanding of individual and contextual factors associated with recurrent hospital admissions involving malnutrition.

\section{Methods}

\section{Study setting}

Secondary data analysis was conducted retrospectively on observational data from 2002 to 2015 of recurrent pediatric ward admissions to Kilifi County Hospital (KCH) in Kenya, including household data from a demographic surveillance. Kilifi County is located in the Coastal region of Kenya (shown in Fig. 1); it is a semi-arid area with subsistence farming being the main economic activity. $\mathrm{KCH}$ is the main referral hospital in Kilifi County with $\sim 4000$ pediatric admissions to $\mathrm{KCH}$ per year. $\mathrm{KCH}$ is in the middle of the Kilifi Health and Demographic Surveillance System (KHDSS) and captures resident children's inpatient details at $\mathrm{KCH}$ pediatric ward. The KDHSS was set to monitor vital demographic indicators, mortality, migration and fertility in the area predominantly served by $\mathrm{KCH}$.

The KHDSS covers 15 administrative locations and 40 sub-locations. The KHDSS area has approximately 280,000 residents. Data are collected three times a year from households to record deaths, migration and birth events. Mortality and morbidity events captured at the hospital are integrated with the population register [14]. The linkage of the surveillance data and admissions data is done real time with the matching of individuals at the point of admissions as explained in detail elsewhere [14]. The data is then deidentified by assigning a unique identifier and a corresponding person identifier for the KHDSS residents.

\section{Study participants}

For this analysis, we included data from children admitted in $\mathrm{KCH}$ from April 2002 to December 2015, aged between 3 months and 5 years, residents of KHDSS who had more than one admission event. We excluded trauma events and children with a missing discharge outcome. The follow-up of the children started after the initial admission until the last admission before reaching 5 years of age (Additional file 1).

\section{Outcome and explanatory variables}

The primary outcome of interest was the number of malnutrition-related readmissions. Malnutrition was defined using Weight for Height Z-score (WHZ) $<-2$ or the presence of oedema at admission. Oedema is a clinical sign of undernutrition which may include swelling of the feet and skin [15]. The WHZ was calculated using the 2006 WHO child growth standards [16]. The weight of the children during admission was done using an electronic scale (Seca, Birmingham, UK) that has a weekly check for consistency $[5,17]$. The heights of the children were measured with a stadiometer (Seca 215, Birmingham, UK) [18] except for children with less than 2 years and those who could not stand whose length was measured using a standard calibrated board.

The explanatory variables were selected with the guidance of the UNICEF malnutrition conceptual framework [1]. Child level variables, i.e. socio-demographic, anthropometric and clinical were selected as the immediate causes and the environmental variables as the underlying 


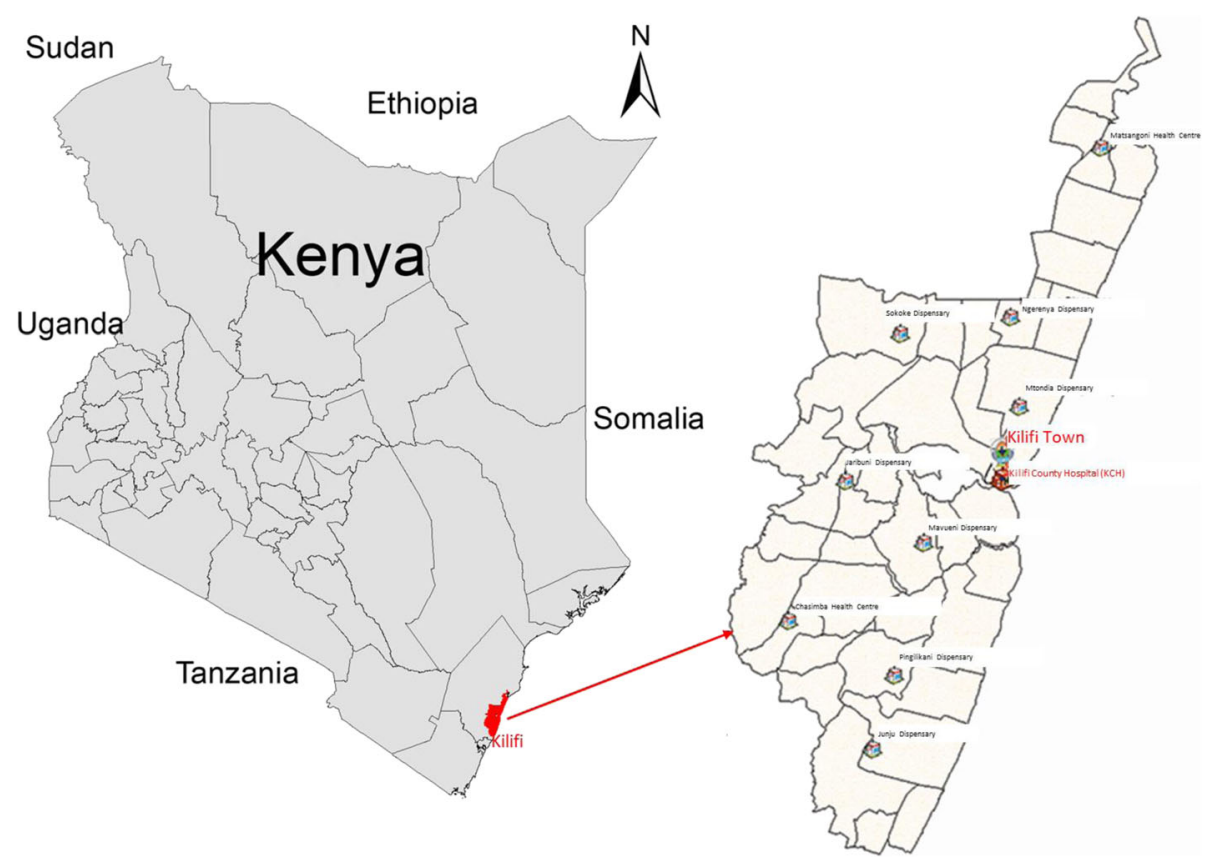

Fig. 1 KHDSS Facilities. a The Kenyan map, showing the location of KHDSS, $\mathbf{b}$ The location of the KHDSS and the different dispensaries and the main county hospital

causes of malnutrition. We generated a composite variable for severe disease, defined as a child admitted with either gastroenteritis, Lower respiratory tract infection (LRTI), blood or Cerebrospinal fluid (CSF) culture positive, malaria and fever or meningitis [5]. The composite variable was generated from several severe co-morbidities that a child had during the readmission times. Environmental predictor variables were extracted from the Moderate Resolution Imaging Spectroradiometer (MODIS) under National Aeronautics and Space Administration's (NASA's) remote-sensing using the MODISTools and MODIS package in R๑ version 3.3.2 [19-21]. Estimates from Enhanced Vegetation Index (EVI) and Rainfall raster files were interpolated from values of the four nearest raster cells of each admission coordinate provided [12]. Additional covariates associated with malnutrition morbidity were identified by a total-sets analysis based on a generalised linear regression model and also those that have a biologically plausible relationship [22].

\section{Data quality}

A team of field workers were trained on using the KHDSS surveillance system to do the matching of the data on real-time admissions. Each field worker has access to the web-based system following the matching procedures of the patients explained elsewhere [14]. Qualified medical and clinical officers enter the history and clinical examination of the patients in the web-app system after matching has been done by the field workers. Data quality checks on clinical measurements are implemented to ensure values entered into the system are within the normal biological range. The database has a daily backup, and each event or record entered into the system has a unique event identifier and an audit trail.

\section{Statistical methods}

Temporal exploratory data analysis

To explore the temporal patterns, the data were defined as a regular time series data using monthly time points of counts of malnutrition-related admissions. Augmented Dickey-Fuller unit-root was used to test for stationarity; the null hypothesis is there is no seasonality trend in the model. The stationary series was then examined on the AutoCorrelation Function (ACF) for the MA lags, and Partial Auto-Correlation Function (PACF) for the AR lags. This was followed by fitting combined Autoregressive integrated moving average (ARIMA) and the seasonal component to form the SARIMA models detailed in Additional file $2[19,23]$.

Seasonality was determined using the ACF and PCF with pointwise confidence intervals based on Bartlett's formula. The Portmanteau test was used to confirm the significance of the seasonality. The Portmanteau test null hypothesis is that there is no serial correlation using the white noise under Pearson's Chi-Square statistic [24]. This was followed by fitting a Seasonal ARIMA (SARIMA) model. 


\section{Spatial exploratory analysis}

The Global Moran Index was used to estimate the measure of sub-location spatial autocorrelation or randomness based on the observed cases of malnutrition admission. A distance matrix of the sub-locations centroids was used to calculate the Moran's index [25, 26]. The Moran's Index was calculated based on the central limit theory on the distribution of the index, which states that as the sample size increases the index tends to a normal distribution. The Moran Index is interpreted depending on the three outcomes; $I>E(I)$ shows a positive autocorrelation implying clustering and $I \approx 0$ shows no spatial autocorrelation and $I<E(I)$ shows a negative autocorrelation implying a dispersed neighbouring value, where $E(I)$ is the normally distributed population mean.

A window scanning over space was done comparing the observed versus the expected over the country using the SaTScan software. SaTScan applies the Kulldorff spatial scan statistic which imposes a circular window and calculates the likelihood of observing the events inside and outside the study area. The circle with the maximum likelihood is defined as the most likely cluster [27, 28]. This helps to determine local heterogeneity and clustering of malnutritionrelated repeated admission to hospital to isolate the hot and cold spots in Kilifi County.

\section{Bivariate analysis}

Our outcome was defined as the counts of malnutritionrelated admissions, reflecting the burden and distribution of malnutrition and pattern of hospital utilisation. The data had a repeated event structure for the individuals over time. For the bivariate analysis, panel data tabulations were applied to investigate the between-individual variation and within-individual variation. This helps to understand the individual systematic difference and differences between individuals over time.

Repeated measures analysis of covariance (ANCOVA) was applied to compare the differences between the continuous predictors and the outcome.

\section{Inferential statistics}

The malnutrition related morbidity was observed to follow a negative binomial distribution. Negative binomial is a generalisation of the Poisson distribution used to provide better epidemiological estimates of factors associated with malnutrition with repeated data $[29,30]$. Secondly, the variance of the malnutrition-related admissions was higher than the mean, so a negative binomial distribution fitted our count data well [29, 31, 32]. Negative binomial spatialtemporal regression was applied to identify the spatial and temporal pattern of malnutrition admissions in Kilifi. The hypothesis tested was that readmission to hospital with malnutrition has spatial and temporal structure due to the individual level, and environmental covariates that underlie the risk. The count of admissions of the individuals was used as the temporal component and age was used as the exposure variable on our model. An autoregressive time series model was used to select the order for the temporal component to use in the spatial-temporal model.

Multivariable analysis was done using non-spatial, spatial and spatial-temporal models for admission specific and environmental variables with sub-location information.

\section{Model fit}

We used the adapted Stochastic Partial Differential Equations (SPDE) in Integrated Nested Laplace approximation (INLA) for the spatial-temporal model, and the Markov chain Monte Carlo (MCMC) with Metropolis-Hastings algorithms approaches in OpenBUGS software from Medical Research Council (MRC) Biostatistics Unit [33] for spatial models using a Bayesian approach. STATA $13^{\text {тm }}$ was used to fit the multilevel models using Maximum Likelihood estimation. The advantage of Bayesian models over the MLE models is the combination of the prior information with the data through Bayes theorem to obtain posterior distributions. Convergence of the Bayesian Markov Chain Monte Carlo approach was monitored using the trace plots. For model comparison and best fit, we used the Deviance Information Criterion (DIC), where the smaller of the DIC was considered better taking into account the Pd. The $\mathrm{pD}$ is the difference of posterior mean deviance and deviance of posterior means which penalises for the parameters in the model $[34,35]$.

\section{Results}

Over the 14 years, 2821 children under- 5 years had at least two admissions in $\mathrm{KCH}$, totalling 6375 admission events including the initial admission. Malnutritionrelated admissions were identified in 1054/2821 (37\% of children ever admitted) of the children. Of the 1054 children ever admitted with malnutrition, $76 \%$ (percentage within) of their re-admissions were malnutritionrelated (Table 1). This shows that there are children who sometimes had a malnutrition re-admission and at other times a non-malnutrition related re-admission. Additionally, of the children with readmissions, $31.6 \%$ of the children had a positive malaria re-admission, and $74 \%$ of their re-admissions were reported to had malaria.

\section{Temporal exploration}

The temporal analysis was fitted for monthly data for the period from April 2002 to December 2015. The time series autoregression order 1 , integration order 0 and moving-average1 forming the ARIMA $(1,0,1)$.

The AutoCorrelation Function (ACF) and Partial autocorrelation Function (PACF) of the transformed series 
Table 1 Panel characteristics of the study population (Child Level Variables) for the period between 2002 and 2015

\begin{tabular}{|c|c|c|c|c|}
\hline & & Overall & & \\
\hline & & Total Individuals & Overall Admissions (\%) & Between N (\% between; $\%$ within) ${ }^{a}$ \\
\hline Gender & Male & 2821 & $3637(57.0)$ & $1583(56.1 ; 100)$ \\
\hline & Female & & $2738(43.0)$ & $1238(43.9 ; 100)$ \\
\hline Age group & $3-23$ months & 2821 & $3846(60.4)$ & $2060(73.0 ; 81.9)$ \\
\hline & 24-60 months & & $2527(39.7)$ & $1519(53.9 ; 74.6)$ \\
\hline Malnutrition & NO & 2821 & $4509(70.7)$ & $2282(80.9 ; 88.8)$ \\
\hline & YES & & $1866(29.3)$ & $1054(37.4 ; 75.5)$ \\
\hline Severe diseases & 0 & 2821 & $2616(41.4)$ & $1709(60.6 ; 66.1)$ \\
\hline & 1 & & $3434(53.9)$ & $2160(76.6 ; 71.5)$ \\
\hline & 2 & & $307(4.8)$ & $284(10.1 ; 48.9)$ \\
\hline & 3 & & $18(0.3)$ & $17(0.6 ; 47.1)$ \\
\hline Severe anaemia & NO & 2807 & $5695(93.6)$ & $2741(97.7 ; 96.0)$ \\
\hline & YES & & $389(6.4)$ & $309(11.0 ; 57.1)$ \\
\hline Hypoglycaemia & NO & 2587 & $5173(99.1)$ & $2572(99.4 ; 99.5)$ \\
\hline & YES & & $48(0.9)$ & $45(1.7 ; 62.5)$ \\
\hline Malaria & NO & 2805 & $4731(78.2)$ & $2375(84.7 ; 90.5)$ \\
\hline & YES & & $1321(21.8)$ & $885(31.6 ; 74.1)$ \\
\hline Diarrhoea & NO & 2821 & 4992 (78.5) & $2559(90.7 ; 85.6)$ \\
\hline & YES & & $1366(21.5)$ & $1055(37.4 ; 59.8)$ \\
\hline Menengitis & NO & 2821 & 6295 (99.2) & 2815 (99.8;99.3) \\
\hline & YES & & $53(0.8)$ & $50(1.8 ; 50.7)$ \\
\hline LRTI & NO & 2821 & 4435 (69.9) & $2441(86.5 ; 81.9)$ \\
\hline & YES & & 1913 (30.1) & $1285(45.6 ; 63.9)$ \\
\hline Gastroenteritis & NO & 2821 & $5391(84.9)$ & $2642(93.7 ; 89.8)$ \\
\hline & YES & & $957(15.1)$ & $772(27.4 ; 58.3)$ \\
\hline Transfused & NO & 2819 & $5969(94.1)$ & $2768(98.2 ; 95.8)$ \\
\hline & YES & & $376(5.9)$ & $302(10.7 ; 55.1)$ \\
\hline Blood Culture & NO & 2795 & 5901 (96.3) & $2779(99.4 ; 96.9)$ \\
\hline & YES & & $227(3.7)$ & $208(7.4 ; 48.7)$ \\
\hline CSF Culture & NO & 950 & 1185 (98.9) & $942(99.2 ; 99.8)$ \\
\hline & YES & & $13(1.1)$ & $12(1.3 ; 81.9)$ \\
\hline
\end{tabular}

NB: YES means positive test result for a given test, Between $\mathrm{N}$ tells us how many children had the specific characteristic of interest, $\%$ between tells us the fraction of the Total Individuals that had the characteristic, \% within tells us the fraction of the re-admissions a child had the specified characteristic. Malnutrition (YES) WHZ $<-2$ or Oedema, Severe anemia (YES)- Hemoglobin $\leq 5 \mathrm{~g} / \mathrm{dl}$, Hypoglycaemia (YES) - Blood glucose $<3.0 \mathrm{mmol} / \mathrm{l}$, Malaria (YES) - parasite by microscopy fever, Meningitis (YES)- Final discharge diagnosis of meningitis; LRTI (Yes) - presence of Lower respiratory tract infections - Cerebrospinal fluid. ${ }^{a}$ - one-way tabulation of counts of between and within individuals in repeated admissions data (panel data)

using data from 2002 to 2015 showed peaks at different lag periods as shown in "Additional file 3".

Significant serial correlation (seasonality) of months between May and July were observed for children admitted with malnutrition as shown in Fig. 2. This was confirmed with a significant $(p<0.001)$ Portmanteau test for white noise. Different peaks are observed, but in general, the malnutrition-related admissions decline over the period. The tables in "Additional file 3" shows the monthly counts of malnutrition admissions and mortality for the period between 2002 and 2015 .

\section{Spatial exploration}

A Global Moran's I of $0.1(p$-value $<0.001, \mathrm{sd}=0.029)$ was observed showing a positive autocorrelation implying clustering. Based on the calculated Global Morans Index, we rejected the zero spatial autocorrelation hypothesis. The Kulldorff spatial scan statistic using SaTScan showed hotspots in the Northern and Southern areas of Kilifi urban area where the main hospital is based. Areas closer to the main hospital and Kilifi town were observed as cold spots of malnutrition-related admissions as shown in Fig. 2. Four of the five hot spots 

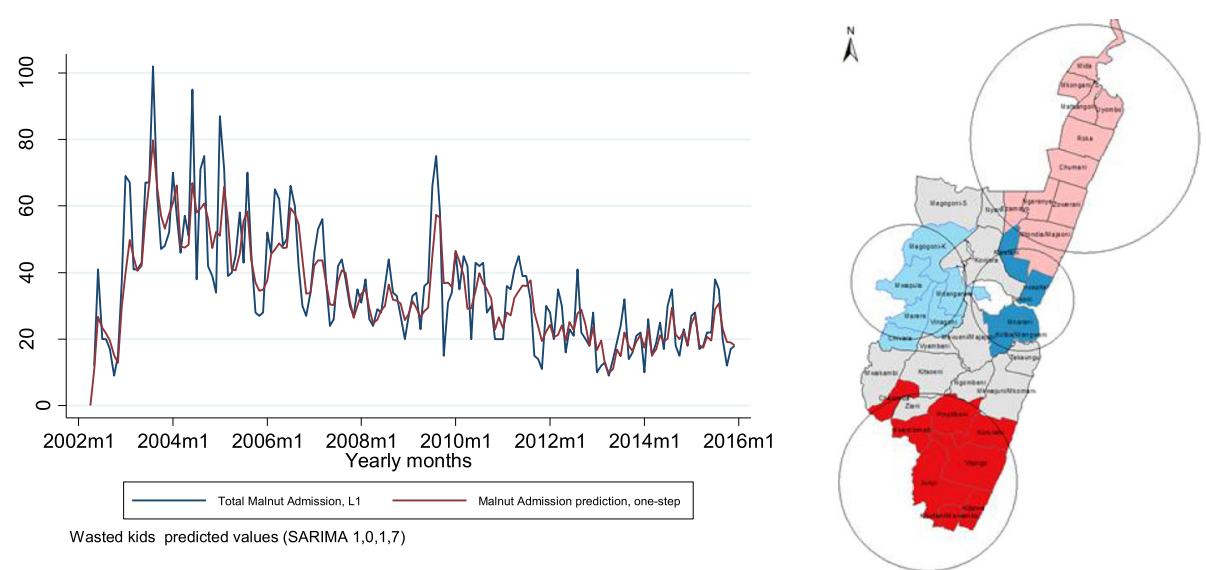

Fig. 2 Time Series and Hotspot analysis results. a time series plot for monthly malnutrition recurrent admissions. b Hotspots and Coldspots of Malnutrition admissions between 2002 and 2015 in KHDSS

and cold spots were significant, the cluster (Mwapula, Marere, Magogoni-K, Mdangarani, Vinagoni, Chivara) was the one observed not to be significant for malnutritionrelated admissions. The temporal clustering of malnutritionrelated admissions (shown in "Additional file 3"), kept on shifting over the periods but similar regions were observed.

The age of the child at re-admission was used as the offset variable, and the count of malnutrition-related admissions was used as the temporal variable for more epidemiological intuitive results. The codes of the Integrated Nested Laplace approximation (INLA) approach and Multilevel modelling are in "Additional file 4".

As shown on Table 2 three models were fitted, a multilevel model adjusting for the sub-location random effects, a spatial model and the spatial-temporal model using nested Laplace approximations which have better computational capability over the Markov Chain Monte Carlo approach
(MCMC) [36]. The estimated mean coefficients are reported in Table 2 with a 95\% confidence interval based on the Maximum Likelihood Estimation (MLE) approach and 95\% credible intervals for the Bayesian approach.

In general, the spatial-temporal models had a lower DIC compared to non-spatial models but with extratemporal parameters. Bayesian spatial-temporal model using R-INLA was the model with the best fit with a $\mathrm{DIC}=10,656.28(\mathrm{pD}=28.41)$ which was the lowest compared to the spatial model which had a DIC $=10$, $982.20(\mathrm{pD}=21.46)$.

The spatial-temporal model showed consistent results with the non-spatial model except for the severe disease which changed. The final spatial-temporal negative binomial model was considered the "model of best fit" which caters well for over-dispersion and spatial-temporal confounding.

Table 2 Non-spatial and spatial multivariable negative binomial models adjusted for duration of admission

\begin{tabular}{|c|c|c|c|c|}
\hline \multirow[t]{2}{*}{ Variables } & \multicolumn{2}{|l|}{ Multi-level model } & \multirow{2}{*}{$\begin{array}{l}\text { Spatial Structured and Unstructured } \\
\text { Random Effects Model (INLA) } \\
\text { Mean }(95 \% \mathrm{Cr} . \mathrm{I})\end{array}$} & \multirow{2}{*}{$\begin{array}{l}\text { Spatial Structured and Unstructured } \\
\text { and Temporal Random Effects Model } \\
\text { Mean }(95 \% \mathrm{Cr} . \mathrm{I})\end{array}$} \\
\hline & Coefficients (95\% C.I) & $p$-value & & \\
\hline \multicolumn{5}{|l|}{ Location level variables } \\
\hline EVI ( & $0.17(-0.54 ; 0.88)$ & 0.644 & $0.07(-0.58 ; 0.70)$ & $0.04(-0.58 ; 0.66)$ \\
\hline Rainfall (mm) & $0.03(-0.03 ; 0.09)$ & 0.350 & $0.03(-0.02 ; 0.08)$ & $0.03(-0.02 ; 0.08)$ \\
\hline \multicolumn{5}{|l|}{ Child level variables } \\
\hline Gender: Male & $-0.15(-0.25 ;-0.04)$ & 0.006 & $-0.13(-0.22 ;-0.04)$ & $-0.13(-0.22 ;-0.04)$ \\
\hline \multicolumn{5}{|l|}{ Severe Disease } \\
\hline 1 & $0.41(0.30 ; 0.52)$ & $<0.001$ & $0.16(0.06 ; 0.25)$ & $0.19(0.10 ; 0.28)$ \\
\hline 2 & $0.43(0.18 ; 0.69)$ & 0.001 & $0.07(-0.16 ; 0.29)$ & $0.15(-0.07 ; 0.37)$ \\
\hline 3 & $0.89(0.06 ; 1.71)$ & 0.036 & $0.56(-0.15 ; 1.25)$ & $0.53(-0.16 ; 1.18)$ \\
\hline Total Number of Admissions & $0.20(0.17 ; 0.23)$ & $<0.001$ & $0.2(0.17 ; 0.22)$ & $0.07(0.04 ; 0.11)$ \\
\hline $\mathrm{DIC}(\mathrm{pD})$ & & & $10982.20(21.46)$ & $10656.28(28.41)$ \\
\hline
\end{tabular}


Adjusting for the spatial-temporal effects and other factors, males were less likely to have malnutrition readmission $(\mathrm{RR}=0.88(\mathrm{Cr} . \mathrm{I}=0.80-0.96)$ compared to the females. In the non-spatial model, the increase in the number of severe diseases increased the risk of malnutrition readmission. On the same note, the spatial-temporal effects, the levels 2 and 3 of severe diseases were insignificant. The environmental variables were not significantly associated with malnutrition re-admission in both the multilevel and spatial-temporal models.

Posterior temporal estimates of readmission were displayed on structured maps shown in Fig. 3 after running the spatial-temporal model. This shows the overall hot spots and cold spots of malnutrition readmission. Over the years, using Kuldorf Statistics ("Additional file 3"), the hotspots were consistent in the north and south of the creek. The readmission hotspots and cold spots were stable until the 6th readmission. The instability in the other readmission events could be due to a few children with these readmission events. Some sub-locations close to the Kilifi town and Kilifi County Hospital were identified as hotspots of readmission with malnutrition after adjusting for covariates. Areas with darker red have a higher risk of admission with malnutrition, and blue shades indicate a lower risk of a malnutrition admission. The plots in Appendix 7 ("Additional file 3") shows that the parameters of the model fitted well since they follow a Gaussian distribution; thus the mean and the mode are equal.

\section{Discussion}

This study describes the spatial-temporal admission of a group of children with repeated malnutrition-related readmissions to Kilifi County Hospital admissions between 2002 and 2015. A seasonality of malnutrition-related readmissions was observed, with peaks occurring in July when the rainy season was mainly observed. Emelda et al. reported a significant decline in malaria admissions at many hospitals in Kilifi. They reported this was observed against a background of elevating or regular non-malaria admissions and impervious to long-term rainfall throughout the surveillance period [37]. In Somalia, Kinyoki et al. observed a clear seasonal variation in wasting for children below 5 years due to variations in climate, food security, and infectious diseases. The peaks of malnutrition were observed during the dry season and were reported to have an elevated effect for the rainy season [37]. This can be used to explain the peaks of malnutrition-related admissions in Kilifi county hospital during the rainy season. However, the environmental factors were not associated with malnutrition readmissions.

Severe diseases, days of admission and rainfall were fit as covariates with malnutrition readmission as the outcome on our model. Severe disease was defined as the presence of either gastroenteritis, Lower respiratory tract infection (LRTI), blood and Cerebrospinal fluid (CSF) culture positive, positive malaria test examined by microscopy and fever or meningitis. In Somalia and Malawi, infections and geographical factors, including

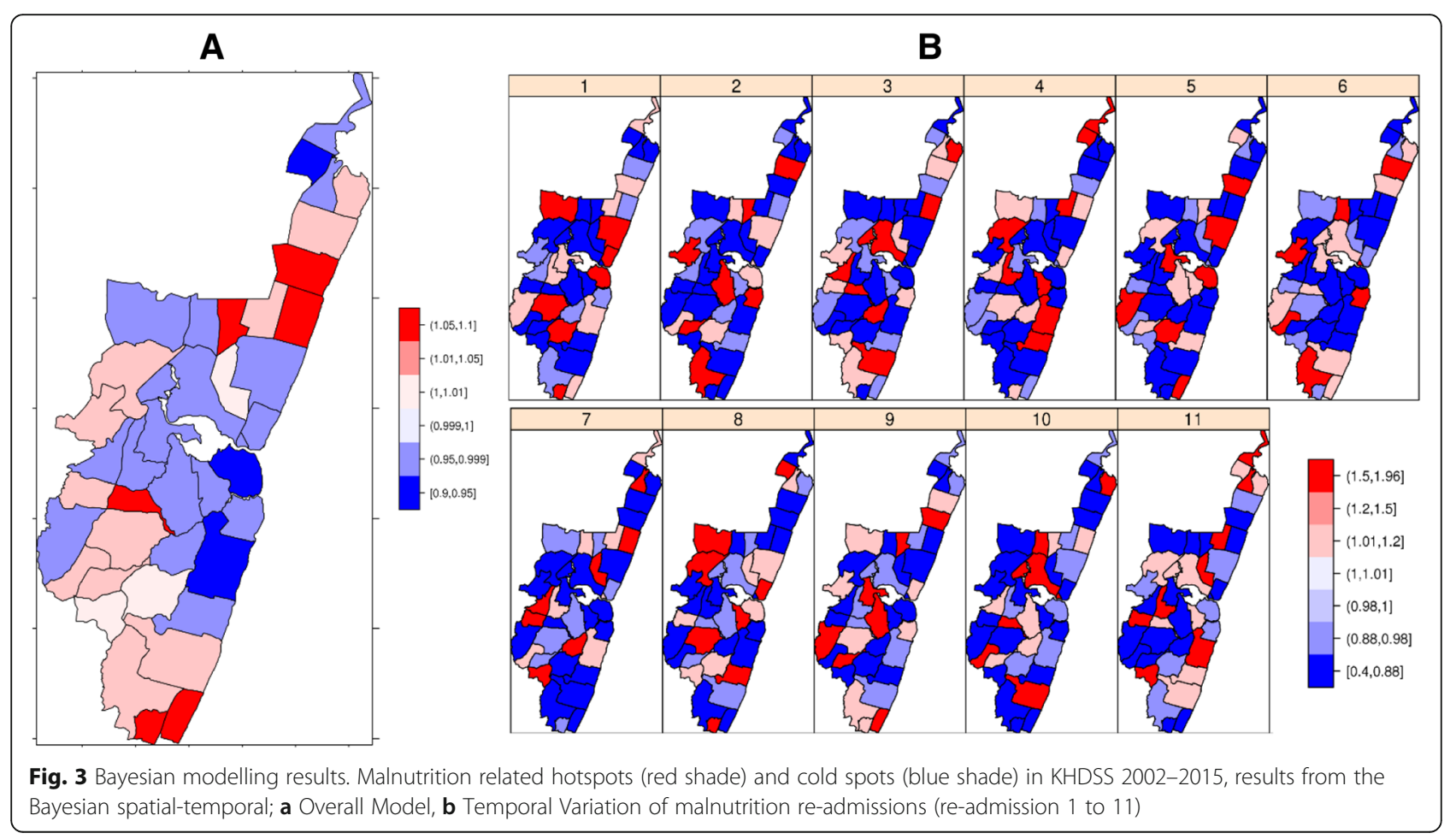


the Enhanced Vegetation Index were observed as critical drivers of malnutrition [13, 38]. Similarly, in our analysis children with a higher number of severe diseases were associated with an increased risk of the admission involving malnutrition. Contrary to the Somalia model, the EVI did not affect the risk of a malnutrition re-admission in the spatial-temporal model. However, overall, the mean EVI value $(0.18)$ in Somalia was lower than the EVI value in Kilifi, Kenya (0.39).

In contrast to EVI, rainfall seasonality was associated with malnutrition admission which is similar to the time series analysis done by Karuri et al. that reported a seasonality during rainfall peaks for malaria admissions in the Kenya Coast [39]. This was different from a longitudinal malnutrition study done in Ethiopia where acute malnutrition did not have a significant seasonality [40]. Here, we combined both the spatial and temporal random effects and the environmental variables in our modelling approach. Thus, malnutrition could be possibly explained by the infections that occur during rainy seasons like diarrhoea or malaria.

\section{Conclusion}

Sabrina et al. in 2014 review recommended the importance of combining spatial and temporal components in understanding the compounded phenomenon of malnutrition [11]. Though, with the different recommendations of spatial models to get an improved understanding of malnutrition; some of them require higher computing resources for an imbalanced and large dataset. In our model, we utilise a spatial-temporal approach which shows the importance of space and time in understanding the risk factors of malnutrition-related morbidity.

This study demonstrates that it is feasible to map episodes of repeated admission to hospital with malnutrition related morbidity at high spatial resolutions. The work has also utilised recently developed statistical tools together to develop spatial-temporal models that converged rapidly without loss of predictive accuracy. The contribution of infections provides a better understanding of the drivers of repeated admissions with malnutrition as a co-morbidity.

\section{Additional files}

Additional file 1: Appendix 1. Flow diagram of data inclusion and exclusion. (DOCX $22 \mathrm{~kb}$ )

Additional file 2: Appendix 2. Time series and Bayesian model derivation. (PDF $144 \mathrm{~kb}$ )

Additional file 3: Appendix 3. Monthly malnutrition related admissions in Kilifi County Hospital between 2002 and 2015. Appendix 4.

Autocorrelation Function (ACF) and Partial autocorrelation function

(PACF) of the SARIMA model. Appendix 5. Shifting hotspots and colsdpots from 2002-2015 using Kulldorff statistics in SaTScan. Appendix 7. Negative Binomial Bayesian fit Diagnostic Plots. (DOCX $4710 \mathrm{~kb}$ )
Additional file 4: Appendix 6 . $R$ code for environmental data extraction and fitting the spatial temporal model. (DOCX $28 \mathrm{~kb}$ )

\section{Abbreviations}

ACF: Autocorrelation Function; ANCOVA: Analysis of Covariance; AR: Auto Regressive; ARIMA: AutoRegressive Integrated Moving Average; CSF: Cerebrospinal fluid; DIC: Deviance Information Criterion; EVI: Enhanced Vegetation Index; INLA: Integrated Nested Laplace Approximation; KCH: Kilifi County Hospital; KHDSS: Kilifi Health and Demographic Surveillance System; LRTI: Lower Respiratory Tract Infection; MCMC: Markov Chain Monte Carlo; MLE: Maximum Likelihood Estimation; MODIS: Moderate Resolution Imaging Spectroradiometer; PACF: Partial autocorrelation function; SARIMA: Seasonal AutoRegressive Integrated Moving Average; SPDE: Stochastic Partial

Differential Equations; UNICEF: United Nations Children's Fund; WHZ: Weight for Height Z Scores

\section{Acknowledgements}

We would like to acknowledge Prof James Berkley who was supportive in article development and KEMRI-Wellcome Trust for the provision of access to the data. David Amadi for the support during data management process.

\section{Authors' contributions}

KM analyzed and interpreted the data and wrote the manuscript. EM advised on the study design and analysis plan and was a major contributor in writing the manuscript. Both authors read and approved the final manuscript.

\section{Funding}

This work was supported through the DELTAS Africa Initiative. The DELTAS Africa Initiative is an independent funding scheme of the African Academy of Sciences (AAS)'s Alliance for Accelerating Excellence in Science in Africa (AESA) and supported by the New Partnership for Africa's Development Planning and Coordinating Agency (NEPAD Agency) with funding from the Wellcome Trust [grant 107754/Z/15/Z- DELTAS Africa Sub-Saharan Africa Consortium for Advanced Biostatistics (SSACAB) programme] and the UK government. The views expressed in this publication are those of the author(s) and not necessarily those of AAS, NEPAD Agency, Wellcome Trust or the UK government.

\section{Availability of data and materials}

The codes and MODIS data used and during the current study are available in the Github repository, under Additional file 4 or the online repository (https://github.com/Keniajin/BMC_nutrition_2019_spatial_temporal). The hospital admission datasets analyzed during the current study are not publicly available because it contains admission data of patient but are available from the corresponding author on reasonable request.

Figures and the Appendix supplementary files are of my own except Fig. 1a shape file. The shape file for the Kenyan map is from GADM and the license is on https://gadm.org/license.html [accessed 25 April 2019]. The license states that "GADM license The data are freely available for academic use and other non-commercial use. Redistribution, or commercial use, is not allowed without prior permission. Using the data to create maps for academic publishing is allowed".

\section{Ethics approval and consent to participate}

Ethical approval under clearance certificate number M1611104 was received from the Human Research Ethics Committee of the University of the Witwatersrand. Consent to participate not applicable.

\section{Consent for publication}

Not applicable.

\section{Competing interests}

The authors declare that they have no competing interests. 
Received: 25 April 2018 Accepted: 22 May 2019

Published online: 04 June 2019

\section{References}

1. Black RERE, Allen LHLH, Bhutta ZAZA, Caulfield LELE, de Onis M, Ezzati M, et al. Maternal and child undernutrition: global and regional exposures and health consequences. Lancet. 2008;371(9608):243-60 /01/22. 2008 Jan 19.

2. Black RE, Victora CG, Walker SP, Bhutta ZA, Christian P, de Onis M, et al. Maternal and child undernutrition and overweight in low-income and middle-income countries. Lancet. 2013;382(9890):427-51 2013/06/12.

3. Forouzanfar MH, Alexander L, Anderson HR, Bachman VF, Biryukov S, Brauer $\mathrm{M}$, et al. Global, regional, and national comparative risk assessment of 79 behavioural, environmental and occupational, and metabolic risks or clusters of risks in 188 countries, 1990-2013: a systematic analysis for the Global Burden of Disease Study 2013. Lancet. 2015;386(10010):2287-323.

4. Sunguya BFP, Koola Jl, Atkinson S. Infections associated with severe malnutrition among hospitalised children in East Africa. Tanzan Health Res Bull. 2006;8(3):189-92.

5. Bejon P, Mohammed S, Mwangi I, Atkinson SH, Osier F, Peshu N, et al. Fraction of all hospital admissions and deaths attributable to malnutrition among children in rural Kenya. Am J Clin Nutr. 2008;88(6):1626-31 2008/12/10.

6. Institute IFPR, Haddad L, Hawkes C, Emorn U, Achadi E, Ahuja A, et al. Global nutrition report 2015: actions and accountability to advance nutrition and sustainable development. 2015;

7. Man WD-C, Man WD-C, Weber M, Weber M, Palmer A, Palmer A, et al. Nutritional status of children admitted to hospital with different diseases and its relationship to outcome in the Gambia, West Africa. Tropical Med Int Health. 1998;3(8):678-86.

8. Bhutta ZA, Ahmed T, Black RE, Cousens S, Dewey K, Giugliani E, et al. What works? Interventions for maternal and child undernutrition and survival. Lancet. 2008;371(9610):417-40.

9. Gewa CA, Yandell N. Undernutrition among Kenyan children: contribution of child, maternal and household factors. Public Health Nutr. 2012;15(6):1029-38

10. Masibo PK, Makoka D. Trends and determinants of undernutrition among young Kenyan children : Kenya Demographic and Health Survey ; 1993 , 1998, 2003 and 2008-2009. 2017;15(9):1715-27.

11. Marx S, Phalkey R, Aranda-Jan CB, Profe J, Sauerborn R, Höfle B, et al. Geographic information analysis and web-based geoportals to explore malnutrition in sub-Saharan Africa: a systematic review of approaches. BMC Public Health. 2014;14(1):1189.

12. Fletcher-Lartey SM, Caprarelli G. Application of GIS technology in public health: successes and challenges. Parasitology. 2016;143(4):401-15.

13. Kinyoki DK, Berkley JA, Moloney GM, Kandala N-B, Noor AM. Predictors of the risk of malnutrition among children under the age of 5 years in Somalia. Public Health Nutr. 2015:18(17):1-9.

14. Scott JAG, Bauni E, Moisi JC, Ojal J, Gatakaa H, Nyundo C, et al. Profile: the Kilifi health and demographic surveillance system (KHDSS). Int J Epidemiol. 2012;41(April):650-7.

15. Golden MN, Golden B, Jackson A. Albumin and nutritional Oedema. Lancet. 1980 Jan;315(8160):114-6.

16. Leroy J. ZSCORE06: Stata module to calculate anthropometric z-scores using the 2006 WHO child growth standards; 2011.

17. Ngari MM, Fegan G, Mwangome MK, Ngama MJ, Mturi N, Scott JAG, et al. Mortality after inpatient treatment for severe pneumonia in children: a cohort study. Paediatr Perinat Epidemiol. 2017;31(3):233-42.

18. Ngari MM, Thitiri J, Mwalekwa L, Timbwa M, Iversen PO, Fegan GW, et al. The impact of rickets on growth and morbidity during recovery among children with complicated severe acute malnutrition in Kenya: a cohort study. Matern Child Nutr. 2018;14(2):e12569.

19. Tuck SL, Phillips HRP, Hintzen RE, Scharlemann JPW, Purvis A, Hudson LN. MODISTools - downloading and processing MODIS remotely sensed data in R. Ecol Evol. 2014;4(24):4658-68.

20. Mattiuzzi M, Jan Verbesselt, Tomislav Hengl, Anja Klisch, Forrest Stevens, Steven Mosher, et al. Acquisition and processing of MODIS product. 2016.

21. R Core Team. R: A language and environment for statistical computing. Vienna: R Foundation for Statistical Computing; 2018. https://www.R-project. org/.

22. Miller AJ. Subset selection in regression. 2nd Edi. Isham V, editor. Florida: Chapman \& Hall/CRC; 2002. 238 p.

23. Coghlan A. A little book of $R$ for time series release $0.2 ; 2017$.

24. Yaffee RA. A review of Stata versions 9 and 10 time series and forecasting capability. J Stat Softw. 2007;23(1):1-8.
25. Gittleman JL, Kot M. Adaptation: statistics and a null model for estimating phylogenetic effects. 1990;39(3):227-241.

26. Paradis E, Claude J, Strimmer K. A \{PE\}: analyses of phylogenetics and evolution in $\{R\}$ language. Bioinformatics. 2004;20:289-90.

27. Coleman MM, Coleman MM, Mabuza AMA, Kok G, Coetzee M, Durrheim DNDN, et al. Using the SaTScan method to detect local malaria clusters for guiding malaria control programmes. Malar J. 2009;8(1):68.

28. Hjalmars U, Kulldorff M, Gustafsson G, Nagarwalla N. Childhood leukaemia in Sweden: using GIS and a spatial scan statistic for cluster detection. Stat Med. 1996;15(7-9):707-15.

29. Musenge E, Vounatsou P, Kahn K. Space-time confounding adjusted determinants of child HIV/TB mortality for large zero-inflated data in rural South Africa. Spat Spatiotemporal Epidemiol. 2011;2(4):205-17.

30. Rogers J. Analysing recurrent events: a review of statistical methodology and future directions, with application to major trials in heart failure outline motivation conventional analyses examples; 2014.

31. Sartorius BKD, Kahn K, Vounatsou P, Collinson MA, Tollman SMSM, Hammer $\mathrm{G}$, et al. Young and vulnerable: spatial-temporal trends and risk factors for infant mortality in rural South Africa (Agincourt), 1992-2007. BMC Public Health. 2010;10(1):645

32. Thomsen JL, Parner ET. Methods for analysing recurrent events in health care data. Examples from admissions in Ebeltoft health promotion project. Fam Pract Adv Access. 2006;23(4):407-13.

33. Spiegelhalter D, Thomas A, Best N, Lunn D. OpenBUGS user manual, version 3.0. 2. MRC biostat unit, Cambridge; 2007.

34. Neelon B, Ghosh P, Loebs PF. A spatial Poisson hurdle model for exploring geographic variation in emergency department visits. J R Stat Soc A Stat Soc. 2013;176(2):389-413.

35. Spiegelhalter DJ, Best NG, Carlin BP, van der Linde A, Spiegelhalter DJ, Best NG, Carlin BP, van der Linde A. Bayesian measures of model complexity and fit. J R Stat Soc Ser B (Statistical Methodol). 2002;64(4):583-639.

36. Rue H, Martino S, Chopin N. Approximate Bayesian inference for latent Gaussian models by using integrated nested Laplace approximations. J R Stat Soc Ser B (Stat Methodol). 2009;71(2):319-92.

37. Okiro EA, Hay SI, Gikandi PW, Sharif SK, Noor AM, Peshu N, et al. The decline in paediatric malaria admissions on the coast of Kenya. Malar J. 2007;6:151 2007/11/17.

38. Kandala N-B, Magadi MA, Madise NJ. An investigation of district spatial variations of childhood diarrhoea and fever morbidity in Malawi. Soc Sci Med. 2006;62(5):1138-52.

39. Karuri SW, Snow RW. Forecasting paediatric malaria admissions on the Kenya coast using rainfall. Glob Health Action. 2016;9:29876.

40. Joosten KFM, Hulst JJM, Antwi S, et al. Prevalence of malnutrition in pediatric hospital patients. Curr Opin Pediatr. 2008:20(5):590-6.

\section{Publisher's Note}

Springer Nature remains neutral with regard to jurisdictional claims in published maps and institutional affiliations.

\section{Ready to submit your research? Choose BMC and benefit from:}

- fast, convenient online submission

- thorough peer review by experienced researchers in your field

- rapid publication on acceptance

- support for research data, including large and complex data types

- gold Open Access which fosters wider collaboration and increased citations

- maximum visibility for your research: over $100 \mathrm{M}$ website views per year

At $\mathrm{BMC}$, research is always in progress.

Learn more biomedcentral.com/submissions 